\title{
Using Satellite Laser Ranging to measure ice mass change in Greenland and Antarctica
}

\author{
Jennifer A. Bonin ${ }^{1}$, Don P. Chambers ${ }^{1}$, Minkang Cheng ${ }^{2}$ \\ ${ }^{1}$ College of Marine Science, University of South Florida, Tampa, FL, 33701, USA \\ $5 \quad{ }^{2}$ Center for Space Research, University of Texas at Austin, Austin, TX, 78759, USA \\ Correspondence to: Jennifer A. Bonin (jbonin@mail.usf.edu)
}

\section{Supplemental Information: Inversion Mathematics}

10 We considered two related inversion methods for this work. The first is the technique described in Bonin and Chambers [2013], which constrains the solution using a simple diagonal matrix of process noise. The second, which we ultimately determined to work better for the low-resolution $5 \times 5$ data, uses a more complicated constraint, based on the auto- and crosscorrelations of modeled data within nearby sub-regions. In this section, we will mathematically define both, as well as giving some basic statistics of each, based on a simulation.

Our simulated 'truth' data is a combination of three sets of model output. Over the non-ice land, we use the Global Land Data Assimilation System (GLDAS) model with the Noah land surface model to estimate hydrological mass change (Rodell et al., 2004) (http://disc.sci.gsfc.nasa.gov/hydrology/data-holdings). Over the oceans, the simulation consists of the "cube 92' version of the ECCO2 model's bottom pressure estimates, which are created at daily resolution on a regular $1 / 4^{\circ} \mathrm{x}^{1} 4^{\circ}$ grid (Menemenlis et al., 2008) (ftp://ecco2.jpl.nasa.gov/data1/cube/cube92/lat_lon/quart_90S_90N/PHIBOT.nc/). The monthly version of the Regional Atmospheric Climate Model version 2.3 (RACMO2) (van Meijgaard et al., 2008) is used to simulate the ice mass change over Greenland and Antarctica. All three series are resampled to a $1^{\circ} \mathrm{x} 1^{\circ}$ equirectangular projection. Because RACMO2 only estimates the effects of surface mass balance, but does not include information about glacier melting, it drastically underestimates mass loss in some areas, especially over western Antarctica. To better approximate the true shape of the mass loss, we replace only the RACMO2 trends with the trends of a 100-km Gaussian-smoothed version of the JPL mascon GRACE data (Watkins et al., 2015). The non-linear interannual, annual, and sub-annual portions all remain the original RACMO2 estimates. After combining the ocean, land, and ice portions of the simulation, we add or subtract a uniform-depth layer of water across the oceans at each time, so as to conserve mass over the entire planet. This simulated 'truth' series is known to $1^{\circ} \times 1^{\circ}$ accuracy, and is transformed to limited $5 \times 5$ and $60 \times 60$ spherical harmonics, to represent the 
simulated SLR-like and GRACE-like visions of the 'truth'. These harmonics are re-converted back into grid form for the least squares inversion.

That inversion technique reads in such spatially-limited grids - simulated, GRACE, or SLR - as its observation array, $\mathbf{y}$, 5 where each element in that array refers to a different latitude/longitude grid point. The other needed input is a series of predetermined regions or spatial kernels, covering the entire world. We tested numerous combinations of regions, but the two we found most effective for this problem are a large-region case for use with the simple process-noise constraint technique (Figure S1a), and a smaller-region case for use with the more complex correlated constraint technique (Figure S1b, d, and f). The goal of the inversion is to determine how much mass goes into each region, in order to result in a $5 \times 5$ or $60 \times 60$ view comparable to the input data.
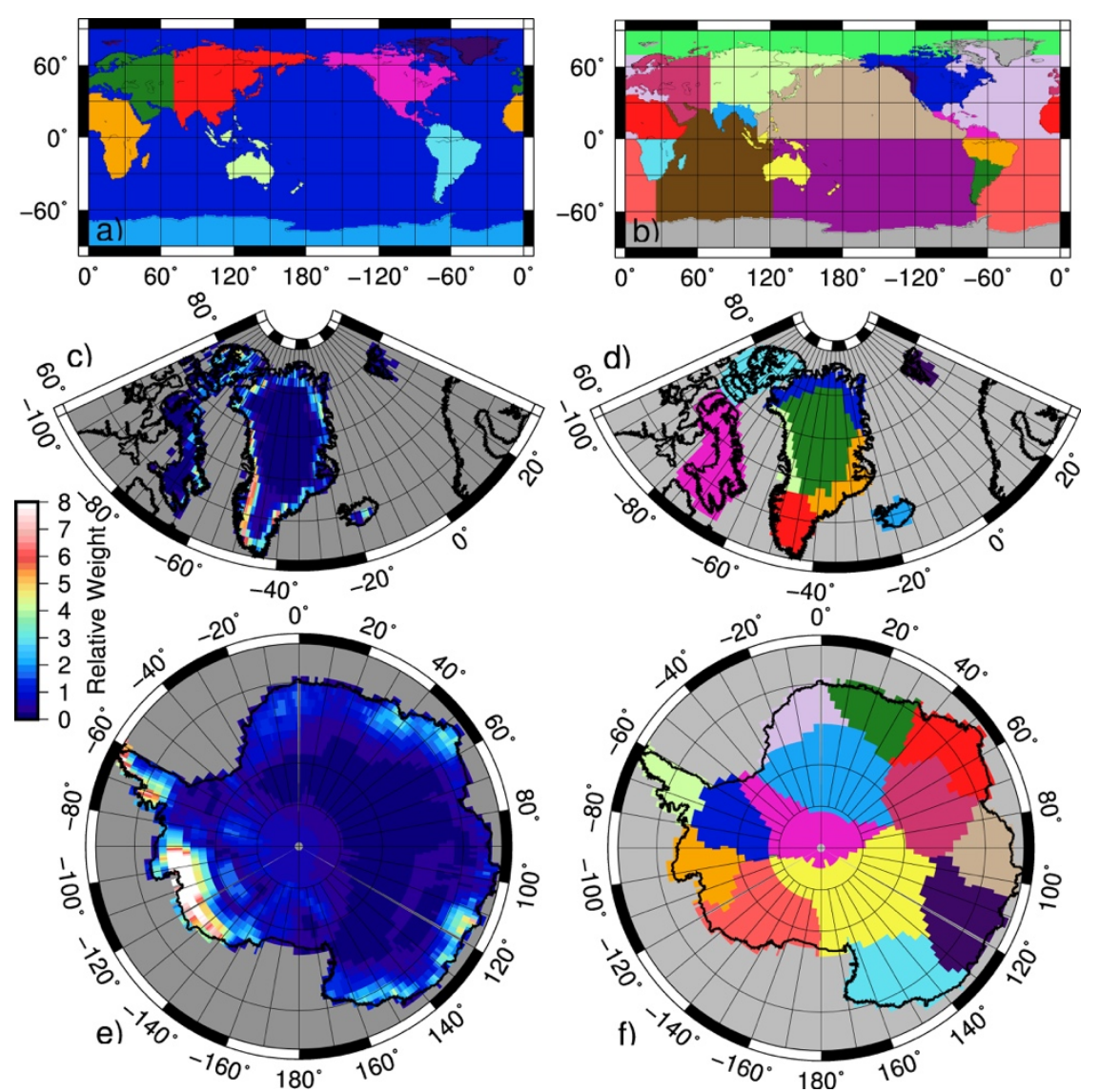

Figure S1: Maps of regions and sub-regions used. (a) shows the large-region layout, while (b), (d), and (f) show the sub-regions of the small-region layout. (c) and (e) are the relative weights applied to the polar regions (which are re-normalized into each smaller sub-region for the small-region cases). The colors in (a), (b), (d) and (f) are arbitrary, merely chosen to visibly separate each area from the others. 
In Bonin and Chambers [2013], we assumed that the mass was uniformly distributed within each region, but in the large regions we are using here, that proved impractical. In this paper, the distribution of mass within many of the regions is still defined as uniform, but each region near Greenland and in Antarctica is weighted by the standard deviation of the RACMO2 series, such that the coastlines are designed to hold most of the mass change signal (Figure S1c and e, normalized for the large-region case). Similarly, six continental regions with the largest annual hydrologic signals are weighted using GLDAS standard deviations.

We wish to create an estimate of the amplitudes, a, which denote the optimal average mass localized into each region at a given time. If $\varepsilon$ is the mismatch between the observations and modeled amplitudes, then what we want to solve can be written as a common least squares observation equation:

$$
\mathbf{y}=(\mathbf{H a}+\varepsilon)
$$

15 The matrix $\mathbf{H}$ defines each weighted region's transformation between its $1^{\circ} \times 1^{\circ}$ gridded shape and a grid limited to the lowerresolution spherical harmonics of the desired maximum degree/order. We can solve for the optimal mass in each region, $\hat{\mathbf{a}}$, by solving the least squares normal equations:

$$
\hat{\mathbf{a}}=\left(\mathbf{H}^{\mathrm{T}} \mathbf{H}\right)^{-1}\left(\mathbf{H}^{\mathrm{T}} \mathbf{y}\right)
$$

20

However, as both Schrama and Wouters [2011] and Bonin and Chambers [2013] determined, without a constraint the least squares technique often misplaces the signal, resulting in the correct sum of mass over a large area, but with it distributed incorrectly internally. Most commonly, this shows itself as a large false positive signal in one sub-region, and an equally large false negative signal in a neighboring one, though the sum of those two sub-regions' signals is correct. To remedy this,

25 Schrama and Wouters [2011] recommended applying an empirically-determined diagonal matrix of process noise, N, as a constraint:

$$
\hat{\mathbf{a}}=\left(\mathbf{H}^{\mathrm{T}} \mathbf{H}+\mathbf{N}\right)^{-1}\left(\mathbf{H}^{\mathrm{T}} \mathbf{y}\right)
$$

30 This works quite well when using 60x60 GRACE data and relatively small regions, as both of the prior papers show. However, using more limited $5 \times 5$ data means using far fewer spherical harmonics as the ultimate input, which prove insufficient for use with large numbers of relatively small regions. In fact, even quite large regions prove too small to solve for accurately. When we tried dividing Antarctica into eastern and western halves, we quickly learned that we could not separate the two correctly, given only $5 \times 5$ data. The same proved true about separating the area around Greenland into more 
than one region. As such, we had no choice but to consider continental-scale regions (as in Figure S1a) instead, when using the simple process-noise constraint technique.

An alternative technique is to separate Antarctica, and separately Greenland, into more pieces, yet tie each of those subregions to the others, and to itself during the surrounding months, via a web of constraints. Mathematically, this can be described by equation S4. We created our constraints from the correlations and variances of the JPL GRACE mascon solutions with GIA removed, using data from 2003-2014.

$$
\hat{\mathbf{a}}_{\mathrm{t}}=\left[\mathbf{H}^{\mathrm{T}} \mathbf{H}+\alpha_{\mathrm{L}}^{2}\left(\mathbf{L}^{\mathrm{T}} \mathbf{L}\right)+\alpha_{\mathrm{C}}^{2}\left(\mathbf{C}^{\mathrm{T}} \mathbf{C}\right)\right]^{-1}\left[\mathbf{H}^{\mathrm{T}} \mathbf{y}+\alpha_{\mathrm{L}}^{2}\left(\mathbf{L}^{\mathrm{T}} \mathbf{L}\right) \overline{\mathbf{a}}_{\mathrm{t}-1}\right]
$$

10

Here, the lag matrix, $\mathbf{L}$, describes the matrix of expected lag-1 auto-correlations for each region, and $\overline{\mathbf{a}}_{\mathrm{t}-1}$ is the value in the grid cell during the previously-computed month. The factor $\boldsymbol{\alpha}_{\mathbf{L}}$ is determined by trial and error; we found a value of approximately 3 produced good $5 \times 5$ results with the simulated 'truth' data. We run the data through twice, forwards and then backwards in time, to iterate and avoid lower accuracy during the first few points in the series.

The cross-correlation matrix, $\mathbf{C}$, contains the expected cross-correlations between each sub-region and every other in the larger region, weighted by the ratio of their variances. This is done similarly but separately for the Greenland and Antarctic areas. $\alpha_{\mathbf{C}}$ is also determined empirically; we found that a value of 1 resulted in the best fit to the simulated 'truth' data in the $5 \times 5$ case.

Equations S5 and S6 show an example of how to set up the $\mathbf{L}$ and $\mathbf{C}$ matrices, for a simple set of three sub-regions $(i, j$, and $k$ ) and the three constraints used to fully connect them. In this example, xcorr $_{i, j}$ denotes the modeled cross-correlation between sub-regions $i$ and $j$, while acorr $_{i}$ is the modeled autocorrelation of sub-region $i$.

$$
\begin{aligned}
& \mathbf{L}=\left[\begin{array}{ccc}
1 & -x \operatorname{corr}_{i, j} & 0 \\
1 & 0 & -x \operatorname{corr}_{i, k} \\
0 & 1 & -x \operatorname{corr}_{j, k}
\end{array}\right] \\
& \mathbf{C}=\left[\begin{array}{ccc}
\operatorname{acorr}_{1} & 0 & 0 \\
0 & \operatorname{acorr}_{2} & 0 \\
0 & 0 & \operatorname{acorr}_{3}
\end{array}\right]
\end{aligned}
$$

Figure S2 demonstrates the relative effectiveness of constraining the inversion via simple process noise, versus constraining it via regional correlations. We computed the average inverted signal over three large regions: all of the Greenland area, all of Antarctica, and the combination of the two. This allows any incorrect internal redistributions of mass from the sub- 
regions to be ignored, while focusing on the bigger, directly-comparable picture. Table S1 lays out each region's trend errors for the different inversions, relative to the 'truth' simulated input.
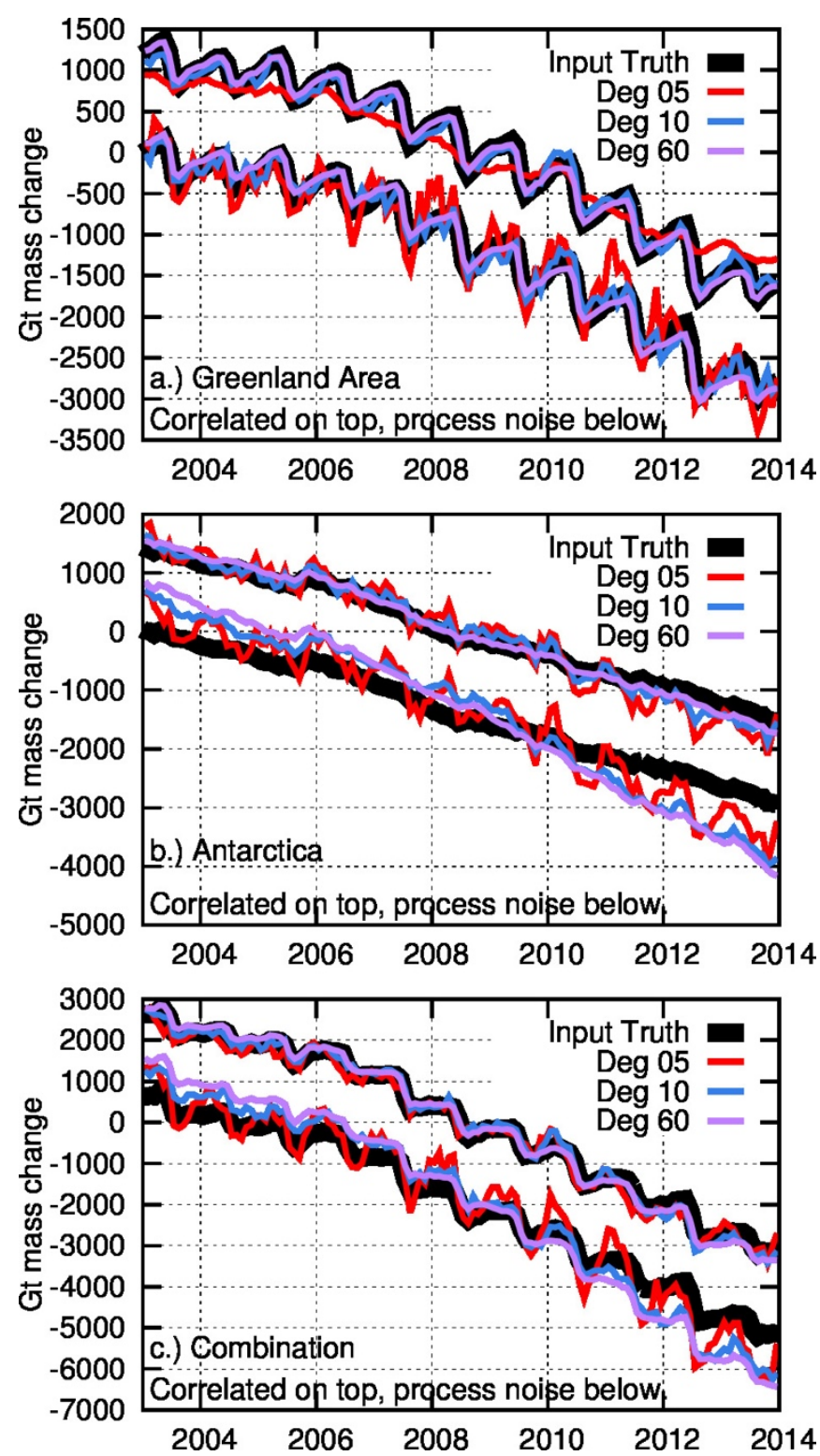

5 Figure S2: Simulated comparison of the large-region, process-noise constrained inversion (top lines in each plot) vs. the smallregion, correlation constrained inversion (bottom lines). Shown are the regional averages over (a) Greenland and the surrounding islands; (b) Antarctica; and (c) the sum of Greenland and Antarctica. The regional average of the input 'truth' is shown in black for comparison. 
We found that in Greenland (Figure S2a), the simple process-noise case worked better than the correlated one, for $5 \times 5$ data. The $5 \times 5$ process-noise constrained inversion results in a lot of jitter, but it recaptures the input simulated seasonal signal and the long-term trend (trend errors are $4.3 \%$ of the full 'truth' trend). When we applied the correlation-based constraint, the $5 \times 5$ inversion was no longer able to pull out the seasonal signal, and the trend had been significantly reduced (trend errors of $17.4 \%$ ). Note that this problem is caused solely by the lack of spatial resolution in the data; with $10 \times 10$ or $60 \times 60$ input data, both problems are be eliminated: the $60 \times 60$ trend errors are $2.8 \%$ when using correlation-based constraints.

\begin{tabular}{|l|c|c|c|}
\hline $\begin{array}{c}\text { Series to Difference, } \\
\text { Relative to Simulated 'Truth' }\end{array}$ & $\begin{array}{c}\text { Greenland } \\
\text { Trend Error } \\
(\%)\end{array}$ & $\begin{array}{c}\text { Antarctica } \\
\text { Trend Error } \\
(\%)\end{array}$ & $\begin{array}{c}\text { Combined } \\
\text { Trend Error } \\
(\%)\end{array}$ \\
\hline process noise $5 \times 5$ & $4.3 \pm 3.1$ & $-30.4 \pm 6.8$ & $-13.0 \pm 3.8$ \\
\hline process noise 10x10 & $-0.5 \pm 1.5$ & $-51.1 \pm 10.3$ & $-25.7 \pm 5.5$ \\
\hline process noise 60x60 & $-7.1 \pm 1.2$ & $-64.5 \pm 6.5$ & $-35.7 \pm 3.2$ \\
\hline correlated constraint 5x5 & $17.4 \pm 4.1$ & $-15.1 \pm 4.7$ & $1.3 \pm 1.6$ \\
\hline correlated constraint 10x10 & $4.5 \pm 1.6$ & $-9.6 \pm 3.1$ & $-2.5 \pm 1.6$ \\
\hline correlated constraint 60x60 & $-2.8 \pm 0.5$ & $-10.2 \pm 1.1$ & $-6.5 \pm 0.8$ \\
\hline high-resolution 60x60 local & $-1.5 \pm 0.3$ & $0.6 \pm 0.5$ & $0.7 \pm 0.5$ \\
\hline
\end{tabular}

Table S1: Simulated inversion differences relative to the 'truth' input, over the three regions. Errors given are purely statistical $\mathbf{9 5 \%}$ confidence levels after fitting for a bias, trend, annual, and semi-annual signals, based on a Monte Carlo simulation of random red noise with the given RMS and lag-1 autocorrelations.

Antarctica (Figure S2b) shows the opposite results: the simple process-noise constraint results in large trend errors, while the correlation-based constraint does a fair job of recovering the simulated input. At $5 \times 5$ resolution, the trend errors increase from $15 \%$ for the constraint-correlated technique, to $31 \%$ for the process-noise-correlated technique. The situation actually grows worse as resolution improves: for $60 \times 60$ data, the errors jump from $10 \%$ to $64 \%$. The reason for this is straightforward and concerns the physical meaning of the computed amplitudes, a, over such huge areas. The different parts of the greater Greenland region tend to vary contemporaneously with each other, so that using a single number to define all of them (in a weighted fashion) will be close to correct, though not perfect. However, Antarctica does not vary so uniformly. Western Antarctica is expected to have lost mass each year recently, while eastern Antarctica is thought to be close to equilibrium and has had periods where the overall mass has likely increased in recent years (Boening et al., 2012). A single value for a cannot represent both situations at once. Either the two sub-regions need to separated - which works for $60 \times 60$ data but is unrealistic with only $5 \times 5$ data - or we need to limit the two halves of Antarctica in different ways. The correlation constraint technique does the latter, and results in a more reasonable estimate for the Antarctic trend because of it.

This leaves us with no technique that works well for all parts of the world. We did attempt to combine equations S3 and S4, so that we use the simple process-noise constraint over Greenland, while at the same time using the correlation constraint 
over Antarctica (not shown). However, we found that the $5 \times 5$ results were still less good than using the correlation constraint everywhere. We hypothesize that the reason we cannot get both the process noise technique's good results in Greenland and the correlation constraint technique's good results in Antarctica at the same time is because the two polar areas are inseparable when looking at only the lowest degree spherical harmonics. Changing the results in one area forces

5 changes in the results of the other.

Because of the inherent connection between Greenland and Antarctica, the only solution we have found is to sum the two and consider that as our final answer (Figure S2c). While our simulation demonstrates that we are unable to accurately separate Greenland from Antarctica at a 5x5 resolution, we can at least confirm that the entire polar land ice mass change can

10 be estimated with reasonable accuracy at an SLR-like spatial resolution. Trend errors over the combined polar regions are estimated at $1.3 \pm 1.6 \%$ of the trend for the correlation-constraint technique, compared to $13 \pm 4 \%$ for the process-noiseconstraint technique. To back out the Greenland area and Antarctic mass changes independently, we would require spherical harmonics which we trust to approximately degree/order 10.

15 This analysis was designed to minimize the errors of a $5 \times 5$ inversion. We found that using the same optimization parameters and regional definitions with $60 \times 60$ data resulted in an over-estimation of the mass trend of $6.5 \pm 0.8 \%$. When using real data, we do not wish this exaggerated trend to appear as part of our comparisons. Instead, when we create 'truth' series for the real-world comparison from the GRACE 60x60 data, we use the simple process-noise technique (equation S3) over each pole separately, and with higher-resolution regions to invert into. Over Greenland, we use the local regions defined in Bonin

20 and Chambers [2015] (excluding the specialized glacier-only basins used there), which include thirteen separate regions in Greenland itself. Over Antarctica, we use the Antarctic division shown in Figure S1f, but with the southern ocean divided into twelve sectors. By computing the inversion locally rather than globally, we are able to achieve excellent similarity to the simulated 'truth' in each region. In Greenland, the trend error is just $-1.5 \pm 0.3 \%$, while in Antarctica it is $0.6 \pm 0.5 \%$. Directly summing the two inverted time-series to estimate the total polar mass loss results in a trend error of only $0.7 \pm$ $250.5 \%$. This is proof that the inversion technique works very well with higher-resolution data. However, we are not able to accomplish the same separation with $5 \times 5$ inputs.

\section{References}

Boening, C., Lebsock, M., Landerer, F. and Stephens, G.: Snowfall-driven mass change on the East Antarctic ice sheet, 30 Geophys. Res. Lett., 39(21), L21501, doi:10.1029/2012GL053316, 2012.

Bonin, J. and Chambers, D.: Uncertainty estimates of a GRACE inversion modelling technique over Greenland using a simulation, Geophys. J. Int., 194(1), 212-229, doi:10.1093/gji/ggt091, 2013. 
Bonin, J. A. and Chambers, D. P.: Quantifying the resolution level where the GRACE satellites can separate Greenland's glacial mass balance from surface mass balance, Cryosph., 9, 1761-1772, doi:10.5194/tc-9-1761-2015, 2015.

van Meijgaard, E., van Ulft, B., van de Berg, W. J., Bosveld, F. C., van den Hurk, B., Lenderink, G. and Siebesma, A. P.: The KNMI regional atmospheric climate model RACMO version 2.1 (KNMI TR-302), Royal Netherlands Meteorological 5 Institute (KNMI), de Bilt, Netherlands., 2008.

Menemenlis, D., J-M. Campin, Heimbach, P., Hill, C., Lee, T., Nguyen, A., Schodlok, M. and Zhang, H.: ECCO2: High resolution global ocean and sea ice data synthesis, Mercat. Ocean Q. Newsl., 31(October), 13-21 [online] Available from: http://www.mercator-ocean.fr/content/download/691/5904/version/1/file/lettre_31_en.pdf\#page=13, 2008.

Rodell, M., Houser, P. R., Jambor, U., Gottschalck, J., Mitchell, K., Meng, C.-J., Arsenault, K., Cosgrove, B., Radakovich,

10 J., Bosilovich, M., Entin*, J. K., Walker, J. P., Lohmann, D. and Toll, D.: The Global Land Data Assimilation System, Bull. Am. Meteorol. Soc., 85(3), 381-394, doi:10.1175/BAMS-85-3-381, 2004.

Schrama, E. J. O. and Wouters, B.: Revisiting Greenland ice sheet mass loss observed by GRACE, J. Geophys. Res., 116(B2), B02407, doi:10.1029/2009JB006847, 2011.

Watkins, M. M., Wiese, D. N., Yuan, D., Boening, C. and Landerer, F. W.: Improved methods for observing Earth's time

15 variable mass distribution with GRACE using spherical cap mascons, J. Geophys. Res. Solid Earth, 120, 1-24, doi:10.1002/2014JB011547.Received, 2015. 